FOLIA

Amazónica

Revista del Instituto de Investigaciones

de la Amazonía Peruana

\title{
COMPORTAMIENTO DEL CADMIO Y OTROS INDICADORES EN SUELO Y ALMENDRA DE CACAO (Theobroma cacao L.), BAJO APLICACIÓN DE COMPOST Y NPK
}

\author{
Nelino FLORIDA ROFNER ${ }^{1}$, Santos JACOBO SALINAS ${ }^{2}$, \\ Tito Felipe GONZALEZ MANRIQUE DE LARA ${ }^{1}$ \\ 1 Universidad Nacional Agraria de la Selva. Av. Universitaria km 1,5 carretera Tingo María, Huánuco. \\ nelinof@hotmail.com \\ 2 Universidad Nacional Hermilio Valdizan. Av. Universitaria Nº61-607, Pillco Marca, Huánuco
}

\section{RESUMEN}

En la última década, las investigaciones advierten elevados niveles de cadmio $\left(\mathrm{Cd}^{2+}\right)$ en suelos y almendra en determinadas zonas del Perú. También, la relación que presenta el $\mathrm{Cd}^{2+}$ con algunos indicadores físicos y químicos del suelo. Por ello, el objetivo fue valuar el efecto del compost y NPK en el $\mathrm{Cd}^{2+}$ del suelo, almendras y la relación con otros indicadores del suelo, en una plantación de cacao CCN-51 (Theobroma cacao L.) en la provincia Padre Abad, región Ucayali-Perú. La investigación utilizó un diseño de bloques completamente aleatorizado (DBCA) con cuatro tratamientos y cuatro repeticiones, donde T1: tratamiento control, T2: compost a razón de $3000 \mathrm{Kg}^{\text {ha-1 }}{ }^{-1}$, T3: NPK en dosis de 84-35-161, y T4 compost a razón de $1500 \mathrm{~kg}$. ha-1 + NPK 42-18-80. Se evaluó $\mathrm{Cd}^{2+}$ disponible en suelo y almendras, el \% de arcilla, \% de materia orgánica (MO), $\mathrm{pH}$, Capacidad de intercambio de cationes (CICe) y $\mathrm{Mg}^{2+}$ intercambiable. Los resultados muestran medias entre 18.5 a $22 \%$ de arcilla, 4.11 a $4.42 \mathrm{de} \mathrm{pH}$, 2.32 a $4.41 \%$ de MO, 7.87 a $9.45 \mathrm{Cmol} / \mathrm{kg}$ de CICe, 0.17 a $0.23 \mu \mathrm{g} \mathrm{g}^{-1} \mathrm{de} \mathrm{Cd}^{2+}$ en el suelo y 0.31 a $0.43 \mu \mathrm{g}$. $\mathrm{g}^{-1}$ en almendras. Se encontró correlación positiva significativa entre los tratamientos y los indicadores $\mathrm{pH}, \mathrm{MO}$ y Cd en almendras y negativa significativa para CICe. Se concluye que la aplicación del compost y NPK presento efecto significativo sobre los indicadores $\mathrm{pH}, \mathrm{MO}$, CICe y $\mathrm{Cd}^{2+}$ en almendras; encontrándose correlación significativa entre la CICe, MO, y $\mathrm{Cd}^{2+}$ en almendra, entre $\mathrm{Mg}^{2+} \mathrm{y} \mathrm{Cd}^{2+}$ en suelo y altamente significativo entre la CICe y 
$\mathrm{Cd}^{2+}$ en suelo. Siendo, T2 (compost) el tratamiento con menores valores de $\mathrm{Cd}^{2+}$ en suelo y almendras.

PALABRAS CLAVE: Cadmio disponible, Cadmio en almendras, Correlación, Compost.

\title{
BEHAVIOR OF CADMIUM AND OTHER INDICATORS IN SOIL AND ALMONDS OF COCOA (Theobroma cacao L.), UNDER APPLICATION OF COMPOST AND NPK
}

\begin{abstract}
In the last decade, research warns of high levels of cadmium $\left(\mathrm{Cd}^{2+}\right)$ in soils and almonds in certain areas of Peru. Also, the relationship that $\mathrm{Cd}^{2+}$ presents with some physical and chemical soil indicators. Therefore, the objective was to evaluate the effect of compost and NPK on the $\mathrm{Cd}^{2+}$ of the soil, almonds and the relationship with other soil indicators, in a cocoa plantation CCN-51 (Theobroma cacao L.) in the Padre Abad province, region Ucayali-Peru. The research used a completely randomized block design (DBCA) with four treatments and four repetitions, where T1: control treatment, T2: compost at a rate of 3,000 Kg.ha-1, T3: NPK at a dose of 84-35-161, and T4 compost at a rate of 1500 kg.ha-1 + NPK 42-18-80; was evaluated $\mathrm{Cd}^{2+}$ available from the soil, and in almonds, $\%$ clay, organic matter (MO), $\mathrm{pH}$ and Cation exchange capacity (CICe). The results show means between 18.5 to $22 \%$ of clay, 4.11 to 4.42 of $\mathrm{pH}, 2.32$ to $4.41 \%$ of MO, 7.87 to $9.45 \mathrm{Cmol} / \mathrm{kg}$ of CICe, 0.17 to $0.23 \mu \mathrm{g}$. $\mathrm{g}^{-1}$ of $\mathrm{Cd}^{2+}$ in the soil and 0.31 to $0.43 \mu \mathrm{g}$. $\mathrm{g}^{-1}$ in almonds. A significant positive correlation was found between the treatments and the $\mathrm{pH}, \mathrm{MO}$ and $\mathrm{Cd}^{2+}$ indicators in almonds and significant negative for CICe. It is concluded that the application of compost and NPK had a significant effect on the $\mathrm{pH}, \mathrm{MO}, \mathrm{CICe}$ and $\mathrm{Cd}^{2+}$ indicators in almonds; finding significant correlation between $\mathrm{CICe}, \mathrm{MO}$, and $\mathrm{Cd}^{2+}$ in almond, between $\mathrm{Mg}^{2+}$ and $\mathrm{Cd}^{2+}$ in soil and highly significant between CICe and $\mathrm{Cd}^{2+}$ in soil. Being, T2 (compost) the treatment with lower values of $\mathrm{Cd}^{2+}$ in soil and almonds.
\end{abstract}

KEYWORDS: Cadmium available, Cadmium in almonds, Correlation, Compost. 


\section{INTRODUCCIÓN}

El cacao (Theobroma cacao L.) es un cultivo de gran importancia económica para Perú (Florida et al., 2018; Arévalo et al., 2016), y según el Ministerio de Agricultura y Riego-MINAGRI (2017) de las 57, 324 toneladas exportadas en el 2016; la región Ucayali aporta con $8 \%$, colocándolo como el cuarto mayor productor del país. Además, el principal mercado de las exportaciones de cacao en grano, es la Unión Europea (UE) (MINAGRI, 2016). Por lo que, la aprobación y entrada en vigor del Reglamento UE No. 488 / 2014 sobre límites máximos de cadmio en cacao y chocolate (0.10 a $\left.0.80 \mu \mathrm{g} \mathrm{g}^{-1}\right)$ a partir del 1 de enero del 2019 (Jiménez, 2015), pone en riesgo nuestras exportaciones hacia la UE.

Investigaciones recientes advierten que los niveles de $\mathrm{Cd}^{2+}$ en suelosy almendra se encuentran por encima de los niveles exigidos por la UE (Florida et al., 2018; Arévalo et al., 2017; Arévalo et al., 2016; Huamaní et al., 2012; Crozier et al., 2012;). Además, se reportan valores altos de $\mathrm{Cd}^{2+}$ en la zona central, zona en que se ubica la región Ucayali (Florida et al., 2018; Tantalean y Huauya, 2017; Huamani et al., 2012; Crozier et al., 2012). Por lo tanto, es necesario generar tecnologías que permitan comprender la dinámica de este metal y disminuir los niveles en almendras.

El origen puede ser natural o antrópico y generalmente se tienen contenidos bajos $\mathrm{de} \mathrm{Cd}^{2+}$ en los suelos, sin embargo, las plantas pueden acumularlo en niveles mayores y en distintas estructuras (Diaz et al., 2018; Casteblanco, 2018; Chávez et al., 2015; Huamani et al., 2012)). También influye la edad de la planta (Sánchez \& Rengifo, 2017). Además, las investigaciones muestran correlación de algunos indicadores del suelo, como el pH, el \% de materia orgánica, el \% de arcilla y la cantidad de $\mathrm{Mg}^{2+}$ y $\mathrm{Zn}$, con el contenido de $\mathrm{Cd}^{+2}$ en suelos y almendras (Florida et al., 2018; Arévalo et al., 2017; Tantalean y Huauya, 2017; Arévalo et al., 2016; Lanza et al., 2016; Huamani et al., 2012). En este contexto, el objetivo del trabajo fue, evaluar el efecto de la aplicación del compost y NPK en el $\mathrm{Cd}^{2+}$ del suelo, almendras y la relación con otros indicadores del suelo, en una plantación de cacao CCN-51 (Theobroma cacao L.) en la provincia Padre Abad, región Ucayali-Perú.

\section{MATERIALES Y MÉTODOS}

\section{ÁREA DE ESTUDIO}

El proyecto se desarrolló entre los meses de enero a diciembre del 2018 en las instalaciones del fundo "Florida \& Cárdenas", ubicada políticamente en la localidad de Nuevo Progreso, distrito y provincia Padre Abad en la región Ucayali - Perú. Las condiciones climáticas en donde se desarrolló este proyecto, presentan una precipitación media anual de $2500 \mathrm{~mm}$, con régimen bimodal, las mayores precipitaciones se producen entre los meses de septiembreabril y la temporada seca desde los meses mayoagosto. Presenta una temperatura media anual de $26,5^{\circ} \mathrm{C}$. Padre Abad, según Holdridge (2000) pertenece al ecosistema de bosque muy húmedo - Premontano Tropical (bmh-PT).

\section{CAMPO EXPERIMENTAL}

Corresponde a una plantación de cacao criollo injertado con yemas del clon CCN-51, de cuatro años de instalación, el distanciamiento entre filas y plantas es de $3 \mathrm{~m}$. Las características de la parcela en el que se instaló el experimento, fisiográficamente se encuentra en terrazas medias no inundable, de origen aluvial antiguo; taxonómicamente corresponde a un suelo Inceptisol (GOREU 2017). Las unidades experimentales son de $9 \times 6 \mathrm{~m}$ que incluyen 
6 plantas de cacao (4 seleccionadas para evaluación) y un área por unidad experimental de $54 \mathrm{~m}^{2}$ hacen un total de $1287 \mathrm{~m}^{2}$.

\section{TRATAMIENTOS}

Se efectuó según el análisis del suelo, la cantidad indicada de compost según ficha técnica. El NPK se utilizó bajo la forma de urea, superfosfato triple y cloruro de potasio en dosis 84-35-161(Tabla 1). La referencia de cálculo es en base al nivel medio de NPK fijado por DS 017-2009-AG (Reglamento de clasificación de suelos por capacidad de uso mayor, para Perú). La aplicación se realizó en contorno a la altura de copa en tres momentos: enero, mayo y setiembre.

Tabla 1. Descripción de los tratamientos (1 111 plan/ha/año)

\begin{tabular}{|c|c|c|c|c|c|}
\hline Tratamiento & $\begin{array}{c}\text { Compost } \\
\text { Kg/ha }\end{array}$ & $\begin{array}{c}\text { Compost } \\
\text { kg/planta }\end{array}$ & $\begin{array}{c}\mathbf{N} \\
\text { g/planta }\end{array}$ & $\begin{array}{c}\mathbf{P} \\
\text { g/planta }\end{array}$ & $\begin{array}{c}\mathbf{K} \\
\text { g/planta }\end{array}$ \\
\hline T1 & 0 & 0 & 0 & 0 & 0 \\
\hline T2 & 3000 & 2,7 & 0 & 0 & 0 \\
\hline T3 & 0 & 0 & 164,36 & 156,3 & 289,9 \\
\hline T4 & 1500 & 1,35 & 82,15 & 78,15 & 144,95 \\
\hline
\end{tabular}

$\mathbf{T} \mathbf{1}=$ Tratamiento control, $\mathbf{T} \mathbf{2}=$ Compost, $\mathbf{T} \mathbf{3}=\mathrm{NPK}, \mathbf{T} \mathbf{4}=$ Compost + NPK.

$\mathrm{N}=$ Nitrógeno,

$\mathrm{P}=$ Fosforo. $\mathrm{K}=$ Potasio

\section{ANÁLISIS DE LABORATORIO}

La investigación inició en enero del 2018 y se realizó el muestreo en octubre, después de la tercera aplicación parcial de los abonos (enero, mayo y setiembre), en las 16 unidades experimentales, tanto para suelos como para almendras de cacao. Para determinar $\mathrm{Cd}^{2+}$ en el suelo, se extrajo 5 sub muestras a $0.2 \mathrm{~m}$ de profundidad de cada unidad experimental, obteniéndose una muestra compuesta de suelo por cada tratamiento y repetición. Además, se evaluó 4 plantas por unidad experimental, de ella se tomó 2 mazorcas al azar de la parte intermedia del árbol en estado de madurez, siguiendo la metodología de Mite (2010). Las muestras fueron derivadas al laboratorio de investigación de la Universidad Nacional Agraria de la Selva. Para el análisis de cadmio disponible del suelo se utilizó como extractante EDTA $0.05 \mathrm{M}$ y en almendras por digestión acida Nítrico Perclórico 4:1 ambos parametros fueron evaluados según Mite (2010). También, se evaluó el \% de arcilla por método de Bouyoucos, MO por Walkley y black, el pH por el método electrométrico 1:1, la CICe y $\mathrm{Mg}^{2+}$ intercambiable por Acetato de amonio, según Bazán (2017), las lecturas se realizaron en espectro de emisión atómica con plasma acoplado inductivamente ICP OES (HORIBA, Ultima Expert).

\section{ANÁLISIS ESTADÍSTICO}

Se utilizó un diseño de bloques completamente aleatorizado DBCA con cuatro tratamientos y cuatro repeticiones, donde $\mathrm{T} 1$ : tratamiento control, T2: compost a razón de $3000 \mathrm{Kg}$. ha ${ }^{-1}$, T3: NPK en dosis de 84-35-161, y T4 compost a razón de $1500 \mathrm{~kg}$. ha-1 + NPK 4218-80. Los datos se sometieron a un análisis de varianza ANVA $(\mathrm{p}<0.05)$ para determinar el efecto del compost y el NPK sobre los diferentes indicadores evaluados y la prueba de Duncan para determinar los subconjuntos homogéneos. Además, se realizó el análisis de correlación de Pearson $(p<0.05)$ con significancia bilateral, para determinar la relación entre las variables. Para el procesamiento, se utilizó el programa IBM SPSS 25.0 


\section{RESULTADO Y DISCUSIÓN}

\section{EFECTO DEL COMPOST Y NPK}

En la Tabla 2, se muestra los indicadores que presentan diferencias significativas al efecto de los diferentes tratamientos, la $\mathrm{MO}$ y $\mathrm{Cd}^{2+}$ en almendras y efectos altamente significativos para pH y CICe. Además, con excepción del tratamiento control, el T2 (compost) seguido del T4 (compost + NPK) presento los menores valores de $\mathrm{Cd}^{2^{*}}$ en almendras (0.36 y $0.39 \mu \mathrm{g}$. $\left.\mathrm{g}^{-1}\right)$, evidenciando la capacidad del componente orgánico en la regulación de la absorción de este metal en almendras de cacao, en comparación con el NPK (fertilizante químico) que presento la media más elevada de $\mathrm{Cd}^{2+}\left(0.43 \mu \mathrm{g}\right.$. $\left.\mathrm{g}^{-1}\right)$.

El NPK es un fertilizante formulado de naturaleza química, con muchos cuestionamientos en la comunidad científica por provocar empobrecimiento de las características biológicas del suelo, disminuyendo la acción de microorganismos que favorecen la absorción de nutrientes para la nutrición de las plantas (López et al., 2007; Chaves et al., 2013). Además, estos fertilizantes pueden aumentar la compactación, la concentración de sales y disminución de materia orgánica y a largo plazo, rendimientos bajos (Orozco et al., 2016). Sin embargo, también se le atribuye mejora en los rendimientos a corto plazo (Abreu et al., 2018), situación que se observa en el caso del T3 (NPK) presento el valor medio mas alto en materia orgánica $4.41 \%$ y el valor medio

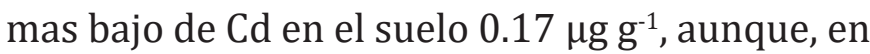
este último no se observa diferencia significativa entre los diferentes tratamientos (Tabla 2), por lo que, no se le puede atribuir efecto positivo del NPK en la reducción del $\mathrm{Cd}^{2+}$ en el suelo.

En general los tratamientos orgánicos (T2 y T4) mostraron efectos significativos en los indicadores $\mathrm{pH}, \mathrm{MO}, \mathrm{CICe}$ y $\mathrm{Cd}^{2+}$ en almendras (Tabla 2). Este comportamiento es contrastado por Abreu et al. (2018); Orozco et al. (2016);

Tabla 2. Indicadores evaluados según tratamiento

\begin{tabular}{|c|c|c|c|c|c|c|}
\hline \multirow{2}{*}{ Indicadores } & \multicolumn{4}{|c|}{ Tratamiento } & \multicolumn{2}{|c|}{ Estadísticos } \\
\hline & T1 & T2 & T3 & T4 & SEM & Sig. \\
\hline$\%$ Arcilla & $20 \pm 2.83$ & $21.5 \pm 1.91$ & $18.5 \pm 1.01$ & $22 \pm 2.58$ & 66,000 & 0,197 \\
\hline $\mathrm{pH}$ & $4.11 \pm 0.09 a$ & $4.39 \pm 0.14 b c$ & $4.26 \pm 0.07 a b$ & $4.42 \pm 0.07 c$ & 0,112 & $0,003^{* *}$ \\
\hline MO \% & $2.32 \pm 0.37 a$ & $4.13 \pm 1.15 b$ & $4.41 \pm 1 b$ & $3.86 \pm 0.64 b$ & 8,618 & $0,019^{*}$ \\
\hline $\begin{array}{c}\mathrm{Mg} \\
\mathrm{Cmol} / \mathrm{kg}\end{array}$ & $1,38 \pm 0.21$ & $1.45 \pm 0.24$ & $1.25 \pm 0.06$ & $1.46 \pm 0.08$ & 0,324 & 0.288 \\
\hline $\mathrm{ClCe} \mathrm{Cmol/kg}$ & $9.45 \pm 0.27 c$ & $8.78 \pm 0.81 b c$ & $7.87 \pm 0.36 a$ & $8.60 \pm 0.4 a b$ & 3,082 & $0,007^{* *}$ \\
\hline $\begin{array}{l}\text { Cd suelo } \\
\mu \mathbf{g ~ g}^{-1}\end{array}$ & $0.23 \pm 0.07$ & $0.25 \pm 0.08$ & $0.17 \pm 0.05$ & $0.22 \pm 0.09$ & 0,067 & 0,481 \\
\hline $\begin{array}{c}\text { Cd Almendras } \\
\mu_{\mathbf{g}^{g-1}}\end{array}$ & $0.31 \pm 0.01$ & $0.36 \pm 0.01$ & $0.43 \pm 0.08$ & $0.39 \pm 0.08$ & 0,034 & $0,046^{*}$ \\
\hline
\end{tabular}

T1=Tratamiento control, T2=Compost, T3=NPK, T4= Compost + NPK. SEM=Error estándar de la media. Las medias seguidas de la misma letra en la línea no difieren entre sí por la prueba de Duncan, $p<0.05$. ${ }^{*}$ presentan diferencias significativas $(p<0.05) .{ }^{* *}$ presentan diferencias altamente significativas $(p<0.01)$. 
Tabla 3. Análisis de correlaciones entre tratamiento e indicadores evaluados

\begin{tabular}{|c|c|c|c|c|c|c|c|c|}
\hline & \multirow{3}{*}{$\begin{array}{c}\text { Arcilla } \\
0.137\end{array}$} & \multirow{3}{*}{$\begin{array}{c}\mathbf{p H} \\
, 583^{*}\end{array}$} & \multirow{3}{*}{$\begin{array}{l}\text { MO } \\
.502^{*}\end{array}$} & \multirow{3}{*}{$\begin{array}{l}\mathbf{M g}^{2+} \\
0.042\end{array}$} & \multirow{3}{*}{$\begin{array}{l}\mathrm{ClCe} \\
-, 540^{*}\end{array}$} & \multirow{3}{*}{$\begin{array}{c}\begin{array}{c}\mathrm{Cd}^{2+} \\
\text { suelo }\end{array} \\
-0.213\end{array}$} & \multirow{3}{*}{$\begin{array}{c}\mathrm{Cd}^{2+} \\
\text { almendra } \\
, 554^{*}\end{array}$} \\
\hline & & & & & & & & \\
\hline Tratamiento & $\begin{array}{l}\text { Correlación } \\
\text { de Pearson }\end{array}$ & & & & & & & \\
\hline & $\begin{array}{l}\text { Sig. } \\
\text { (bilateral) }\end{array}$ & 0.613 & 0.018 & 0.048 & 0.877 & 0.031 & 0.428 & 0.026 \\
\hline & $\mathrm{N}$ & 16 & 16 & 16 & 16 & 16 & 16 & 16 \\
\hline \multirow[t]{3}{*}{ Arcilla } & $\begin{array}{l}\text { Correlación } \\
\text { de Pearson }\end{array}$ & 1 &, $526^{*}$ & 0.156 & 0.458 & 0.253 & 0.429 & 0.293 \\
\hline & $\begin{array}{l}\text { Sig. } \\
\text { (bilateral) }\end{array}$ & & 0.037 & 0.564 & 0.074 & 0.344 & 0.097 & 0.271 \\
\hline & $\mathrm{N}$ & & 16 & 16 & 16 & 16 & 16 & 16 \\
\hline \multirow[t]{3}{*}{ pH } & $\begin{array}{l}\text { Correlación } \\
\text { de Pearson }\end{array}$ & & 1 &, $665^{* *}$ & 0.448 & -0.056 & 0.269 & 0.306 \\
\hline & $\begin{array}{l}\text { Sig. } \\
\text { (bilateral) }\end{array}$ & & & 0.005 & 0.082 & 0.837 & 0.313 & 0.249 \\
\hline & $\mathrm{N}$ & & & 16 & 16 & 16 & 16 & 16 \\
\hline \multirow[t]{3}{*}{ MO } & $\begin{array}{l}\text { Correlación } \\
\text { de Pearson }\end{array}$ & & & 1 & 0.251 & -0.317 & -0.071 &, $582^{*}$ \\
\hline & $\begin{array}{l}\text { Sig. } \\
\text { (bilateral) }\end{array}$ & & & & 0.349 & 0.231 & 0.794 & 0.018 \\
\hline & $\mathrm{N}$ & & & & 16 & 16 & 16 & 16 \\
\hline \multirow{3}{*}{ Mg } & $\begin{array}{l}\text { Correlación } \\
\text { de Pearson }\end{array}$ & & & & 1 &, $522^{*}$ &, $523^{*}$ & -0.142 \\
\hline & $\begin{array}{l}\text { Sig. } \\
\text { (bilateral) }\end{array}$ & & & & & 0.038 & 0.038 & 0.599 \\
\hline & $\mathrm{N}$ & & & & & 16 & 16 & 16 \\
\hline \multirow[t]{3}{*}{$\mathrm{CICe}$} & $\begin{array}{l}\text { Correlación } \\
\text { de Pearson }\end{array}$ & & & & & 1 &, $623^{* *}$ &,$- 533^{*}$ \\
\hline & $\begin{array}{l}\text { Sig. } \\
\text { (bilateral) }\end{array}$ & & & & & & 0.010 & 0.033 \\
\hline & $\mathrm{N}$ & & & & & & 16 & 16 \\
\hline \multirow[t]{3}{*}{$\begin{array}{l}\mathrm{Cd}^{2+} \\
\text { suelo }\end{array}$} & $\begin{array}{l}\text { Correlación } \\
\text { de Pearson }\end{array}$ & & & & & & 1 & -0.284 \\
\hline & $\begin{array}{l}\text { Sig. } \\
\text { (bilateral) }\end{array}$ & & & & & & & 0.287 \\
\hline & $\mathrm{N}$ & & & & & & & 16 \\
\hline
\end{tabular}

*. La correlación es significativa en el nivel 0,05 (bilateral). **. La correlación es significativa en el nivel 0,01 (bilateral). 
Firme et al. (2014) señalan que la aplicación de enmiendas orgánicas puede mejorar las propiedades químicas del suelo, como el $\mathrm{pH}, \mathrm{MO}$ y la CIC. Además, hay un efecto significativo sobre el $\mathrm{Cd}^{2+}$ en almendras; esto se debe a la capacidad que tienen las enmiendas orgánicas en la retención de metales en el suelo, disminuyendo, los factores de movilidad al producir un incremento de los sitios de intercambio, formando complejos estables, la precipitación de minerales y el intercambio iónico y su absorción por las plantas (Tantalean y Huauya, 2017; Cortes et al., 2016; Bravo et al., 2014; Firme et al., 2014). Por lo tanto, los tratamientos con componentes orgánicos T2 y T4, presentan los resultados más favorables por los efectos señalados de la materia orgánica en el pH, MO, CIC y en la retención e inmovilización del $\mathrm{Cd}^{2+}$ disponible del suelo.

\section{CORRELACIÓN DE VARIABLES}

Los resultados de la Tabla 3, muestran correlación positiva significativa entre los tratamientos y los indicadores $\mathrm{pH}, \mathrm{MO} \mathrm{yCd}^{2+}$ en almendras y negativa significativa para CICe. La CICe, considerando los beneficios de la incorporación de enmiendas orgánicas (Abreu et al., 2018; Cortes et al., 2016; Orozco et al., 2016; Firme et al., 2014), se espera tener una correlación positiva, pues en general incrementa los sitios de intercambio, esto puede explicarse, ya que se utilizó tratamientos con fertilizante químico (T3) que presentó los menores valores medios de CICe $(7.87 \mathrm{Cmol} / \mathrm{kg})$, influyendo en la correlación negativa observada. Sin embargo, los tratamientos con compost presentaron los mayores valores (8.78 para T2 y 8.60 para T4) y muestran mayor estabilidad de este indicador evaluado.

En la Tabla 3, también se observa correlación significativa entre la $\mathrm{CICe}, \mathrm{MO}, \mathrm{y} \mathrm{Cd}^{2+}$ en almendra, entre $\mathrm{Mg}^{2+} \mathrm{y} \mathrm{Cd}^{2+}$ en suelo y altamente significativo entre la CICe y $\mathrm{Cd}^{2+}$ en suelo. No se encontró correlación significativa entre el pH y el
$\mathrm{Cd}^{2+}$ en suelos y almendras. Este último resultado, concuerda con los resultados de Florida et al. (2018) en parcelas de socios de la Cooperativa Cacao Alto Huallaga, Huánuco, señala que el pH encontrado no se correlaciona significativamente con $\mathrm{Cd}^{2+}$ del suelo y almendras. También, Huamani et al. (2012) no encontró correlación significativa entre el $\mathrm{Cd}^{2+}$ foliar y él $\mathrm{pH}$, lo mismo ocurre para él \% de arcilla.

Sin embargo, resultados contrarios son reportados por, Arévalo et al. (2016) quien encontró correlación positiva significativa $(\mathrm{p}<0.05)$ entre el $\mathrm{Cd}^{2+}$ y el $\mathrm{pH}$. Además, encontró correlación significativa positiva entre el $\mathrm{Cd}^{2+} \mathrm{y}$ la $\mathrm{MO}, \mathrm{P}, \mathrm{CIC}, \mathrm{Ca}^{2+}, \mathrm{Mg}^{2+} \mathrm{y} \mathrm{K}^{+}$respectivamente, estos últimos coinciden con nuestros resultados.

También, Huamani et al. (2012) en muestras de Huánuco, San Martín y Ucayali encontró correlaciones de cadmio en suelo con el contenido foliar de $\mathrm{P}, \mathrm{Mg}^{2+}$ y $\mathrm{Ca}^{2+}$. Por su parte, Tantalean \& Huauya (2017) en suelo residual encontró correlación positiva con la CIC y negativa entre Cd y el \% de arcilla, mas no encontró correlación para el \% de arcilla en suelo aluvial, lo que sugiere que a mayor \% de arcilla menor será la concentración de cadmio disponible, asumimos que el bajo contenido de arcilla (18.5 T3 a 22\% T4) en nuestra investigación es responsable de que no se encuentre correlación significativa. Por lo tanto, las correlaciones encontradas en la investigación, muestran coincidencias con los resultados de las referencias citadas.

\section{CADMIO EN SUELOS Y ALMENDRAS}

Finalmente, la Tabla 2, muestra los resultados de $\mathrm{Cd}^{2+}$ en suelos (T3=0.17y T2=0.25 $\mu \mathrm{g} . \mathrm{g}^{-1}$ ), encontrándose por debajo de lo establecido por USEPA (2002), estableció como nivel crítico en $0.43 \mu \mathrm{g}$. $\mathrm{g}^{-1}$ de $\mathrm{Cd}^{2+}$ en suelos agrícolas y según Acevedo et al. (2005) se consideran suelos no contaminados por la UE, que estableció un rango de 0 a $1 \mu \mathrm{g}$. $\mathrm{g}^{-1}$. También, los niveles medios de 
$\mathrm{Cd}^{2+}$ en almendras ( $\mathrm{T} 1=0.31$ y $\mathrm{T} 30.43 \mu \mathrm{g} . \mathrm{g}^{-1}$ ) se encuentran en niveles bajos al no superan los límites permisibles de la UE (Reglamento $\mathrm{N}^{\circ} .488$ / 2014), que establece como contenido máximo de $\mathrm{Cd}^{2+}$ en almendras de $0.80 \mu \mathrm{g}$. $\mathrm{g}^{-1}$ (Jiménez, 2015). Además, los niveles de cadmio en almendras son menores a los resultados de las referencias citadas (Florida et al., 2018; Tantalean \& Huauya, 2017; Sánchez \& Rengifo, 2017; Lanza et al., 2016; Chávez et al., 2015), con excepción de los resultados de Arévalo et al. (2016); Sofia y Pablo (2013), que reportan valores medios menores a esta investigación.

\section{CONCLUSIÓN}

- Se encontró diferencias significativas $(p<0.5)$ al efecto de los tratamientos sobre los indicadores $\mathrm{MO} \mathrm{y} \mathrm{Cd}^{2+}$ en almendras y efectos altamente significativos para $\mathrm{pH}$ y CICe. Con excepción del tratamiento control, el T2 (compost) seguido del T4 (Compost + NPK) presento los menores valores de $\mathrm{Cd}^{2+}$ en almendras (0.36 y $0.39 \mu \mathrm{g}$. g- 1 ).

- Los valores medios de $\mathrm{Cd}^{2+}$ en suelos (T3=0.17y $\mathrm{T} 2=0.25 \mu \mathrm{g} . \mathrm{g}^{-1}$ ), es considerado bajo para USEPA (2002) y también para la directiva Kelley de la Unión Europea.

- Los niveles medios de $\mathrm{Cd}^{2+}$ en almendras (T1 $=0.31$ y T3 $0.43 \mu \mathrm{g} . \mathrm{g}^{-1}$ ) es menor que el nivel máximo permitido por la Unión Europea (Reglamento No. 488/2014).

- El $\mathrm{Cd}^{2+}$ en el suelo y almendra presenta correlación significativa $(\mathrm{p}<0.05)$ con la MO, CICe y el $\mathrm{Mg}^{2+}$; no se encontró correlación con el $\mathrm{pH}$ y el $\%$ de arcilla.

\section{BIBLIOGRAFÍA CITADA}

Abreu, C.; Araujo, C.; Rodríguez, J.; Valdivia, Á.; Fuentes, A. \& Pérez, H. 2018. Efecto de la aplicación combinada de fertilizante químico y humus de lombriz en Capsicum annuum. Revista Centro Agrícola. Vol.45(01):52-61. Disponible en: http://scielo.sld.cu/pdf/cag/ v45n1/cag07118.pdf

Acevedo, E.; Carrasco, M.; León, O.; Silva, P.; Castillo, G.; Ahumada, I.; Borie, G. \& González, S. 2005. Informe de criterios de calidad de suelo agrícola. Servicio Agrícola y Ganadero, Chile. $205 \mathrm{pp}$.

Arévalo, G., Arévalo, H., Baligar, V. \& He, Z. 2017. Heavy metal accumulation in leaves and beans of cacao (Theobroma cacao L.) in major cacao growing regions in Peru. Science of The Total Environment. 605-606:792-800.

Arévalo, G. E.; Meyier, E.; Obando, C.; Zúñiga, C. L.; Cesar, O.; Arévalo, H. C. O.; Baligar, V.; Zhenli, He. 2016. Heavy metals in soils of cocoa plantations (theobroma cacao l.) in three regions of peru. Ecología Aplicada, 15(2): 8189.

Bazán, T. R. 2017. Manual de procedimientos de los análisis de suelos y agua con fines de riego. Universidad Nacional Agraria la Molina, Instituto Nacional de Innovación Agraria. LimaPerú. 92 p. Disponible en: http://repositorio. inia.gob.pe/bitstream/inia/504/1/BazanManual_de_procedimientos_de_los.pdf

Bravo, R.; Arboleda, P. \& Martín, P. 2014. Efecto de la calidad de la materia orgánica asociada con el uso y manejo de suelos en la retención de cadmio en sistemas altoandinos de Colombia. Acta Agronómica, vol. 63 (02): 164-174. Disponible en: http://www.redalyc.org:9081/ articulo.oa?id=169930904007

Casteblanco, J. A. 2018. Técnicas de remediación de metales pesados con potencial aplicación 
en el cultivo de cacao. La Granja: Revista de Ciencias de la Vida 27(1):21-35.

Chávez, E.; Stoffella, P.; Mylavarapu, R.; Moyano, B. \& Baligar, V. 2015. Concentration of cadmium in cacao beans and its relationship with soil cadmium in southern Ecuador. Science of The Total Environment. Volume 533(01): 205-214. Chaves, B.; Ortíz, M. \& Ortiz, R. 2013. Efecto de la aplicación de agroquímicos en un cultivo de arroz sobre los microorganismos del suelo. Acta Agronómica. 62 (01): 66-72.

Cortes, P.; Bravo, R.; Martin, P. \& Menjivar, F. 2016. Extracción secuencial de metales pesados en dos suelos contaminados (Andisol y Vertisol) enmendados con ácidos húmicos. Acta Agron. Vol. 65(03): 232-238.

Crozier, J. 2012. Heavy metals in Cocoa. International Workshop on Possible EU regulations on cadmium in cocoaand chocolate products. Nature and Food Quality in the Netherlands (LNV), The European Cocoa Association (ECA) and CAOBISCO.

DECRETO SUPREMO N 017-2009-AG. Aprueban Reglamento de Clasificación de Tierras por su Capacidad de Uso Mayor. http://sinia.minam. gob.pe/normas / aprueban-reglamentoclasificacion-tierras-capacidad-uso-mayor

Díaz, U.; Mendoza, H.; Bravo B. \& Dominguez, V. 2018. Determinación de Cadmio y Plomo en almendras de cacao (Theobroma cacao), proveniente de fincas de productores orgánicos del cantón Vinces. Espirales. 2(15) 77-92. Disponible en: https://doi. org/10.31876/re.v2i15.213

Firme, L.P.; Alvarez, V. F. \& Rodellab, A. A. 2014. Solo contaminado com cádmio: Extratibilidade do metal e cinética química de degradação da matéria orgânica de torta de filtro. Quim. Nova, Vol. 37 (06): 956-963.

Florida, R. N.; Claudio, M. S.; Gómez, B. R. 2018. El pH y la absorción de cadmio en almendras de cacao orgánico (Theobroma cacao L.) en Leoncio Prado, Huánuco, Perú. Folia Amazónica. Vol. 27 (01): 1-8..

Gobierno Regional de Ucayali- GOREU. 2017. Estudio Zonificación Ecológica y Económica de la Región Ucayali, Ordenanza Regional $\mathrm{N}^{\circ}$ 015-2017-GRU-CR publicado en el diario oficial El Peruano el 21 de octubre de 2017. Disponible en: http://ider.regionucayali.gob. pe/

Holdridge, R. L. 2000. Ecología basada en zonas de vida. Quinta reimpresión. Instituto Interamericano de Cooperación para la Agricultura (IICA) San José- Costa Rica, 216p.

Huamani, H. A.; Huauya, M. A.; Mansilla, L. G.; Florida, R. N. \& Neira, G. M. 2012. Presencia de Metales pesados en el cultivo de cacao (Theobroma cacao L.) orgánico. Acta Agronómica. 61(4):339-344.

Jiménez, T. C. S. 2015. Estado legal mundial del cadmio en cacao (Theobroma cacao): fantasía o realidad. Producción + Limpia. 10(01): 89104.

Lanza, J. G.; Churión, P. C.; Liendo, N. J.; López, V. H. 2016. Evaluación del contenido de metales pesados en cacao (teobroma cacao l.) de santa bárbara del zulia, Venezuela. Saber, Universidad de Oriente, Venezuela. Vol. 28, n.o 1: 106-115.

López, M.; López, R.; España, M.; Izquierdo A. \& Herrera L. (2007). Efecto de la fertilización inorgánica sobre la disponibilidad de nutrimentos en el suelo, nivel nutricional de la planta y hongos micorrícicos arburculares en plantaciones de Theobroma cacao. Agronomía Trop. Vol. 57(01). Disponible en: http:// www.scielo.org.ve/scielo.php?script $=$ sci_ arttext\&pid=S0002-192X2007000100005 MINAGRI. 2017. Estudio del cacao en el Perú y en el mundo. Un Análisis de la Producción y el Comercio al 2017. Dirección Estudios Económicos e Información Agraria-DEEIA: $60 \mathrm{pp}$. 
MINAGRI. 2016. Estudio del cacao en el Perú y en el mundo; Situación Actual y Perspectivas en el Mercado Nacional e Internacional al 2015. MINAGRI-DGPA-DEEIA, $86 \mathrm{pp}$

Mite, F.; Carrillo, M. \& Durango, W. 2010. Avances del monitoreo de presencia de cadmio en almendras de cacao, suelos y aguas en Ecuador. Actas XII Congreso Ecuatoriano de la Ciencia del Suelo. Santo Domingo, 17-19 de Noviembre del 2010.

Orozco, C.; Valverde, F.; Martínez, T.; Chávez, B. \& Benavides, H. 2016. Propiedades físicas, químicas y biológicas de un suelo con manzano biofertilizado. Terra Latinoamericana 34: 441456. Disponible en: http://www.scielo.org.mx/ pdf/tl/v34n4/2395-8030-tl-34-04-00441.pdf REGLAMENTO (UE) N. 488/2014. De la comisión de 12 de mayo de 2014, que modifica el Reglamento (CE) N. $-1881 / 2006$ por lo que respecta al contenido máximo de cadmio en los productos alimenticios. Diario Oficial de la Unión Europea, pp 5. Disponible en: https:// www.boe.es/doue/2014/138/L0007500079.pdf

Sánchez, R. \& Rengifo T. 2017. Evaluación del contenido de metales pesados $(\mathrm{Cd}$ y $\mathrm{Pb})$ en diferentes edades y etapas fenológicas del cultivo de cacao en dos zonas del Alto Huallaga, Huánuco (Perú). Rev. de investig. agroproducción sustentable 1(01): 87-94.

Sofia, A. \& Pablo, P. 2013. Determinación de Cadmio en la Almendra de Cacao (Theobroma cacao) de cinco fincas ubicadas en vía San Domingo, Esmeralda [tesis] Pontifica Universidad del Ecuador, facultad de ciencias exactas y naturaleza, Ecuador. 2013: 81 pg. [Citado el 28 de enero 2019]. Disponible en: file:///C:/Users/Usuario/ Downloads/43-1-51-1-10-20170614.pdf

Tantalean, P. E. \& Huauya, R.M. 2017. Distribución del contenido de cadmio en los diferentes órganos del cacao CCN-51 en suelo aluvial y residual en las localidades de Jacintillo y Ramal de Aspuzana. Rev. de investig. agroproducción sustentable 1(2): 69-78. Disponible en: http:// revistas.untrm.edu.pe/index.php/INDESDOS/ article/view/199/0.

United States Environmental Protection Agency (USEPA). 2002. Supplemental guidance for developing soil screening levels for superfund sites. Available from: http://www.epa.gov/ superfund/health/conmedia/soil/ind ex.htm.

Recibido: 14 de marzo de 2018 Aceptado para publicación: 12 de julio de 2018

Esta obra está bajo una Licencia Creative Commons Atribución-NoComercial-SinDerivar 4.0 Internacional. 